\title{
Regeneration of vegetation in manor park in Laskowice (Dąbrowa Forest District)
}

\author{
Dorota Gawenda-Kempczyńska*, Iwona Paszek, Tomasz Załuski
}

\author{
Department of Biology and Pharmaceutical Botany, Nicolaus Copernicus University in Toruń, \\ Ludwik Rydygier Collegium Medicum in Bydgoszcz, \\ M. Skłodowskiej-Curie 9, 85-094 Bydgoszcz, Poland, \\ *e-mail: dgawenda@cm.umk.pl
}

Received: 26 September 2017 / Accepted: 30 October 2017

\begin{abstract}
Laskowice is a village located in Kujawsko-Pomorskie Voivodeship, in Świecie Upland near Świecie. On the edges of the village there is a manor-palace complex established by Franz von Gordon in the first half of the 19th century. Since the palace was destroyed in 1945, the maintenance of the park was ceased. Currently the object is administrated by Dabrowa Forest District.

The aim of the study was to determine the direction of vegetation transformation of the park in the light of lack of use. Lack of human interference within examined object has initiated regeneration processes. At present the vegetation of the park refers to structure and species composition of natural forest plant communities. Phytosociological research carried out in 2015 and 2016 revealed the presence of following associations: Ficario-Ulmetum, Fraxino-Alnetum and Tilio-Carpinetum. Shrub vegetation of Rhamno-Prunetea class develops in places. Non-forest vegetation of meadows (Arrhenatheretum elatioris) and nitrophilous edges (Urtico-Aegopodietum) are present. In case of analyzed object the physionomy and species composition of forest communities differs from typical forms. An attention is paid to a strongly developed layer of brushwood. There is a lack of some characteristic species, alien species are present in treestand and shrub layers. A large share in phytocoenoses of Ficario-Ulmetum and Tilio-Carpinetum has Sambucus nigra and in case of the first association also alien species - Philadelphus coronarius and Symphoricarpos albus. Values of calculated indices of synanthropization confirm the regeneration of forest communities: index of natural character achieves value $\geq 40 \%$ and synanthropization index ranges from $48.78 \%$ for Tilio-Carpinetum to $60.00 \%$ for Fraxino-Alnetum.
\end{abstract}

Key words: forest vegetation, Ficario-Ulmetum, Fraxino-Alnetum, Tilio-Carpinetum, index of natural character, synanthropization index, apophytization index.

\section{Introduction}

The manor park in Laskowice occupies area of 8.84 ha. It is located in Świecie Upland mesoregion near Świecie. It encompasses subdivision 253i within Dąbrowa Forest Division (Regional Directorate of State Forests in Torun), Laskowice Forest Circle, Gródek District. It is located in the northern part of the village by Laskowickie Lake. It is situated on the right side of Park Street that constitutes voivodeship road no 272 linking Laskowice and Jeżewo.
The palace in neo-Gothic style was erected in 18401860 by Franz von Gordon - a Scot, that was the owner of Laskowice since 1828. The park in english style was established in the first half of the 19th century by using natural elements of landscape. The palace was situated on a hill (Fig. 1), with a view extending to the Zamkowe Lake. In the front of the building, from the northern side, there was a courtyard with a fountain and a tennis court. On the north-eastern part manor buildings were erected and vegetable garden was established on the north-western part. The park was dissected by several gravel paths. Only remnants of the palace and a few-storey manor build- 
ing from 1948 has preserved up to the present (Chudecki, 1994; von Weitzel-Zenker, 2002; http://amzpbig.com/ maps/2476_Jaschewo_1910.jpg).

In park's wooded lands apart from alien species mainly native species were planted (Chudecki, 1994; Paszek \& Gawenda-Kempczyńska, 2016). The palace was destroyed in 1945. Since then a maintenance was ceased, that caused gradual naturalizing of the plant cover. At the end of 20th century revitalization actions of the park were taken (Chudecki, 1994), but with no positive effects. The geobotanical research of this area was scarce and it concerned mainly, as in case of the majority of similar objects, dendroflora's inventory with special regard to monumental trees (Didyk, 2013).

Manor parks constitute an essential element of arable landscape of Poland. Some of them are under management of State Forests (Pietrzak \& Zawadka, 2009; WildnerNurek, 2010). At present most of them is not maintained and left for regeneration processes leading to the development of plant communities referring to natural forest communities. However, the majority of carried out research in such objects concerns flora's inventory with special regard to exotic taxa and nature monuments, while phytosociological research is carried out less often (Borowski et al., 2007; Bacieczko \& Wołejko, 2011; Kułak \& Sender,
2011; Fornal-Pieniak \& Ollik, 2013). Therefore the aim of the study herein is a characterization of plant communities for future revitalization of the park. Moreover, an attempt of defining the direction of changes in the light of cessation of use was made, and it is assumed that the vegetation of the park is regenerating.

\section{Methods}

A classical phytosociological method was used for the elaboration of plant communities. The field works were made in 2015 and 2016. Plant communities were determined on a basis of phytosociological relevés by using Braun-Blanquet's method. Sums of average cover values of main elements of plant communities were set out in a synthetic table (according to Dzwonko, 2007). Species were grouped into geographic-historical groups (Mirek et al., 2002; Tokarska-Guzik, 2012). The full phytosociological documentation was deposited in Department of Biology and Pharmaceutical Botany, Ludwik Rydygier Collegium Medicum in Bydgoszcz, Nicolaus Copernicus University in Toruń.

The classification of plant communities as well as species belonging to phytosociological classes were adopted

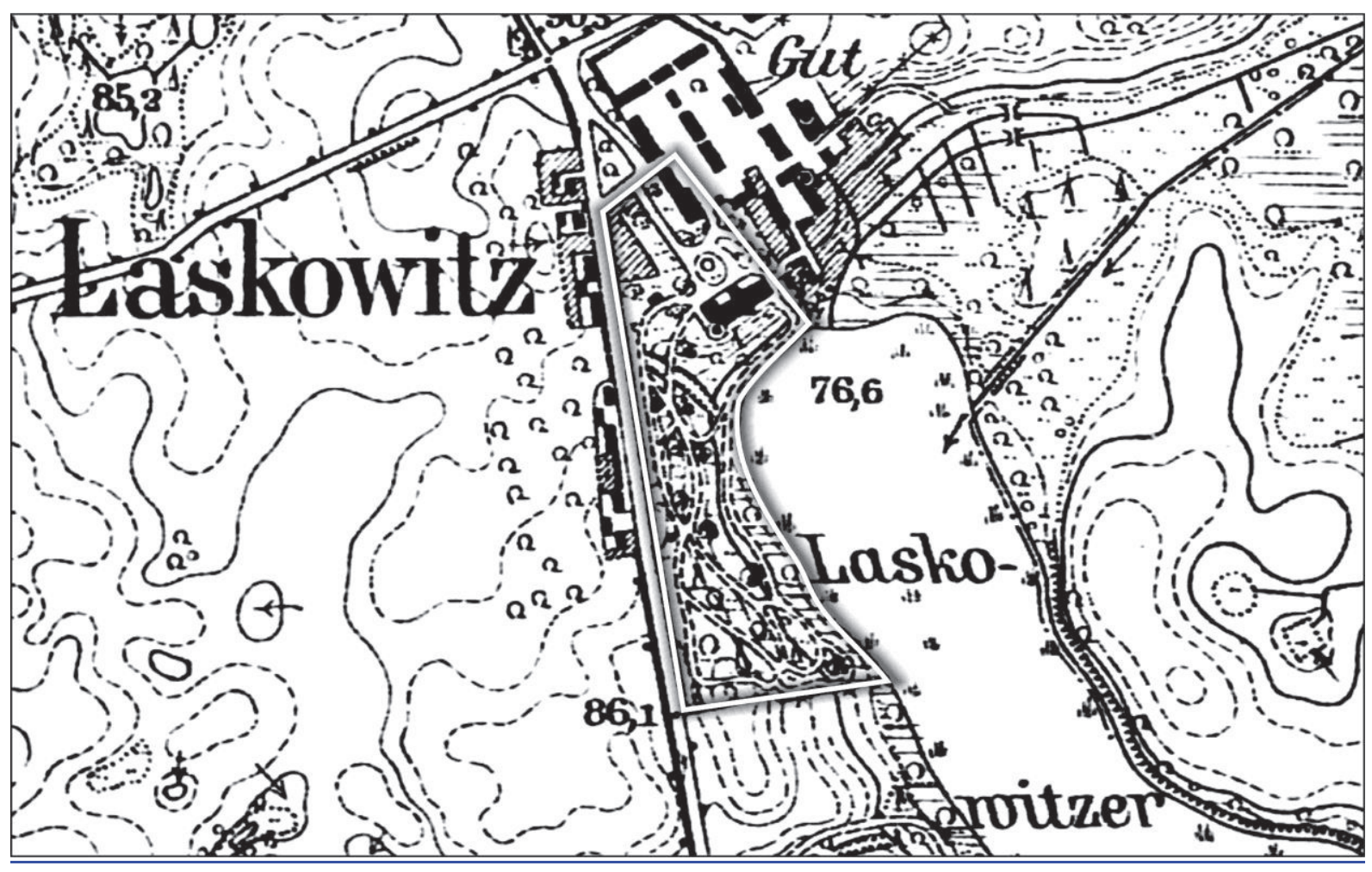

Figure 1. Historical map of the manor park (source: http://amzpbig.com/maps/2476_Jaschewo_1910.jpg). White line - present boundary of the park 


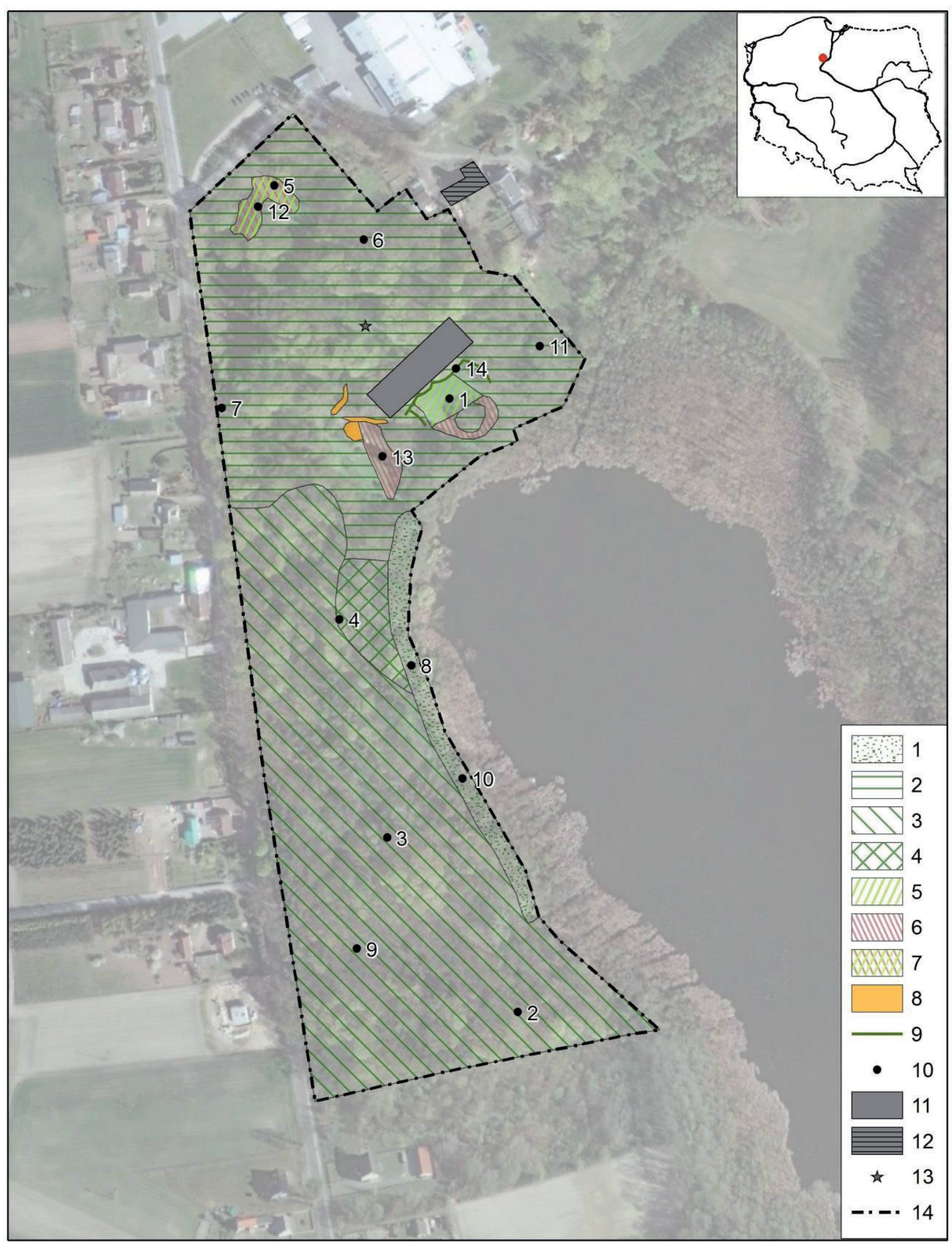

Figure 2. Actual vegetation (explanations: 1 - Fraxino-Alnetum, 2 - Ficario-Ulmetum minoris, 3 - Tilio-Carpinetum typicum 4 Tilio-Carpinetum corydaletosum, 5 - Arrhenatheretum elatioris, 6 - Urtico-Aegopodietum, 7 - mosaic of Arrhenatheretum elatioris and Urtico-Aegopodietum, 8 - Clematis vitalba community, 9 - Parthenocissus inserta and Clematis vitalba community, 10 - localities and numbers of the phytosociological relevés, 11 - ruins of the palace, 12 - preserved manor buildings, 13 - remains of the fountain, 14 - boundary of the park) 
according to Matuszkiewicz (2001), while species nomenclature according to Mirek et al. (2002). The map of real vegetation was performed by using ArcGIS software. A diagram of socio-ecological share of groups was made for each community. Anthropogenic indices (Chmiel, 1993): index of natural character, proper synanthropization index and apophytization index were calculated for each of the plant communities. Natura 2000 sites were determined on a basis of Journal of Laws (Obwieszczenie M.Ś. z dn. 30 października 2014).

\section{Results}

Seven plant community units at level of association or undefined community were noted within the manor park in Laskowice (Fig. 2). Forest communities of QuercoFagetea are dominant there. There are: Fraxino-Alnetum developing in vicinity of the Zamkowe Lake and FicarioUlmetum minoris developing in the northern part of the park, which belong to Alno-Ulmion alliance. Tilio cordatae-Carpinetum betuli which belongs to Carpinion betuli alliance develops in the southern part. Two subassociations were determined - typical Tilio-Carpinetum typicum and moist Tilio-Carpinetum corydaletosum. Non-forest vegetation occupies a small area within the park. It is represented by the Arrhenatheretum elatioris (Molinio-Arrhenatheretea class) and Urtico-Aegopodietum (Artemisietea vulgaris class), dominated by Aegopodium podagraria. Plant communities of Rhamno-Prunetea class: Clematis vitalba community as well as Parthenocissus inserta and Clematis vitalba community have developed along the road and paths near park ruins, on the edges of forest communities.

Fraxino-Alnetum forms over a dozen meters wide belt along the boundary of the Zamkowe Lake. The community was determined on a basis of the occurrence of distinguishing species Alnus glutinosa and Padus avium and more abundant, than in case of Ficario-Ulmetum, share of characteristic species of Fagetalia sylvaticae alliance, including the presence of nitrophilous Impatiens noli-tangere (Table 1). The two-layer treestand is mainly made of Alnus glutinosa, Fraxinus excelsior and Acer pseudoplatanus. Geographically alien Aesculus hippocastanum is present in some places. A shrub layer with a high density $(80 \%)$ is dominated by Sambucus nigra. In this layer there are also present: Padus avium and saplings. Aegopodium podagraria and Galeobdolon luteum are dominant in the middle of vegetation season. Ficaria verna, Anemone ranunculoides, Adoxa moschatellina and Corydalis cava grow in spring.

The treestand of Ficario-Ulmetum is usually monolayer and is characterised by high tree density. Depending on the phytocoenosis, it is comprised of: Fraxinus excelsior, Acer platanoides and Fagus sylvatica with a smaller share of other taxa. Sambucus nigra is dominant in a shrub layer where species not occurring in analyzed phytocoenoses: Symphoricarpos albus and Philadelphus coronarius have also a large share. A presence of Aesculus hippocastanum was also noted in one phytocoenosis. Aegopodium podagraria has a large share in a herb layer, reaching in cover up to $80 \%$ during the middle of the vegetation season. In spring occur: Ficaria verna, Anemone ranunculoides and Gagea minima.

Tilio cordatae-Carpinetum betuli occurs in the southern part of the object. The Carpinion betuli alliance is represented by Tilia cordata, Carpinus betulus and Stellaria holostea. Species characteristic for Fagetalia sylvaticae order are abundant. The treestand is double-layer and it is composed mainly by Tilia cordata. There are also present: Fagus sylvatica, Acer pseudoplatanus, Acer platanoides, Fraxinus excelsior, Betula pendula and alien species: Quercus rubra and Pseudotsuga menziesii. Sambucus nigra is dominant in the shrub layer, similarly as in the case of the rest of forest communities. Lonicera xylosteum and saplings are also present in the majority of phytocoenoses. Galeobdolon luteum, Poa nemoralis and Stellaria holostea occur in the middle of the vegetation season, while in spring: Anemone ranunculoides, Anemone nemorosa and Corydalis cava. A large share of the last of the species mentioned above allowed to distinguish the subassociation Tilio-Carpinetum corydaletosum. Aegopodium podagraria has a large share in phytocoenoses of this community.

Communities like Parthenocissus inserta and Clematis vitalba community as well as Clematis vitalba community, developed near the former palace, along trampled paths. A herb layer with a small cover develops under the dense canopy of Parthenocissus inserta and Clematis vitalba. Meadow species like Arrhenatherum elatius, Carex hirta, Ranunculus acris, Dactylis glomerata, Poa pratensis and Veronica chamaedrys grow aside from saplings and deciduous shrubs like Euonymus europaeus, Fraxinus excelsior and Acer platanoides.

Arrhenatheretum elatioris develops on the slope of the hill, where palace was located, and in northern part of the park. This community occurs in mosaic with Urtico-Aegopodietum. Meadow species of Arrhenatheretalia order: Arrhenatherum elatius, Achillea millefolium and Dactylis glomerata are dominant in phytocoenoses of Arrhenatheretum elatioris. The class of ruderal vegetation Artemisietea vulgaris is represented abundantly, of which the most abundant are: Aegopodium podagraria, Urtica dioica and Glechoma hederacea. The herb layer cover in analyzed phytocoenoses is $100 \%$.

Species of Artemisietea vulgaris class are dominant in phytocoenoses of Urtico-Aegopodietum association, of which the largest share have: Aegopodium podagraria, Urtica dioica and Anthriscus sylvestris. Arrhenatherum 
Table 1. Total cover of species occurring in plant communities (F-A - Fraxino-Alnetum, F-U - Ficario-Ulmetum minoris, T-C - TilioCarpinetum, Par.-Cle. - Parthenocissus inserta and Clematis vitalba community, Arr. - Arrhenatheretum elatioris, U-A Urtico-Aegopodietum) and classification of species into geographical-historical groups ( $\mathrm{Ar}$ - archeophyte, $\mathrm{Kn}$ - kenophyte, $\mathrm{Sp}$ - spontaneophyte, Ap - apophyte)

\begin{tabular}{|c|c|c|c|c|c|c|c|}
\hline Plant community & F-A & $\mathrm{F}-\mathrm{U}$ & $\mathrm{T}-\mathrm{C}$ & Par.-Cle. & Arr. & U-A & \\
\hline Number of releves & 2 & 3 & 4 & 1 & 2 & 2 & $\fallingdotseq$ \\
\hline Area of releves $\left[\mathrm{m}^{2}\right]$ & 100 & 100 & 100 & 24 & 25 & 25 & $5_{0}$ \\
\hline Average cover of tree layer $\mathrm{a}_{1}[\%]$ & 42.5 & 85 & 67.5 & - & - & - & 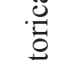 \\
\hline Average cover of tree layer $\mathrm{a}_{2}[\%]$ & 10 & 5 & $<5$ & - & - & - & 㾞 \\
\hline Average cover of shrub layer b [\%] & 80 & 40 & 47.5 & 90 & - & - &. \\
\hline Average cover of herb layer c [\%] & 75 & 83.3 & 80 & 20 & 100 & 100 & \\
\hline Average cover of bryophytes layer [\%] & $<5$ & $<5$ & $<5$ & - & $<5$ & $<5$ & 0 \\
\hline Mean number of species & 36 & 39 & 44 & 16 & 48 & 28 & \\
\hline \multicolumn{8}{|l|}{ ChAll. Alno-Ulmion } \\
\hline Alnus glutinosa $\mathrm{a}_{1}$ & 30 & 2.5 & 2.5 & - & - & - & $\mathrm{Sp}$ \\
\hline Padus avium $\mathrm{b}$ & 2.6 & - & 2.5 & - & - & - & \\
\hline Padus avium $\mathrm{c}$ & 5 & - & 2.5 & - & - & - & \\
\hline Ficaria verna & 77.5 & 90.1 & 0.1 & - & 15 & - & Ap \\
\hline Circaea alpina & 0.1 & - & - & - & - & - & $\mathrm{Sp}$ \\
\hline Festuca gigantea & 0.1 & 0.1 & - & - & - & - & $\mathrm{Sp}$ \\
\hline Gagea lutea & - & 2.6 & 0.1 & - & - & - & $\mathrm{Sp}$ \\
\hline Plagiomnium undulatum $\mathrm{d}$ & 0.1 & 0.1 & - & - & - & - & - \\
\hline \multicolumn{8}{|l|}{ ChAll. Carpinion betuli } \\
\hline Tilia cordata $\mathrm{a}_{1}$ & - & 2.5 & 140 & - & - & - & \\
\hline Tilia cordata $\mathrm{b}$ & - & - & 5 & - & - & - & Ap \\
\hline Tilia cordata c & 0.2 & - & 0.2 & - & - & - & \\
\hline Carpinus betulus $\mathrm{a}_{2}$ & 15 & - & 2.5 & - & - & - & $\mathrm{Sp}$ \\
\hline Stellaria holostea & - & - & 2.5 & - & - & - & $\mathrm{Sp}$ \\
\hline \multicolumn{8}{|l|}{ ChO. Fagetalia sylvaticae } \\
\hline Acer pseudoplatanus $\mathrm{a}_{1}$ & 15 & - & 40 & - & - & - & \\
\hline Acer pseudoplatanus $\mathrm{a}_{2}$ & - & - & 2.5 & - & - & - & $A n$ \\
\hline Acer pseudoplatanus b & 17.5 & 5.1 & 10 & - & - & - & תр \\
\hline Acer pseudoplatanus c & 2.6 & 2.5 & 2.6 & - & 2.5 & - & \\
\hline Fagus sylvatica $\mathrm{a}_{1}$ & - & 87.5 & - & - & - & - & \\
\hline Fagus sylvatica $\mathrm{c}$ & - & 0.1 & - & - & - & - & \\
\hline Ulmus glabra b & - & 15 & - & - & - & - & $\mathrm{Sp}$ \\
\hline Ulmus glabra $\mathrm{c}$ & - & 2.6 & - & - & - & - & \\
\hline
\end{tabular}




\begin{tabular}{|c|c|c|c|c|c|c|c|}
\hline Anemone ranunculoides & 30 & 17.5 & 17.6 & - & - & - & $\mathrm{Sp}$ \\
\hline Galeobdolon luteum & 52.5 & - & 35 & - & - & - & $\mathrm{Sp}$ \\
\hline Impatiens noli-tangere & 2.6 & - & - & - & - & - & $\mathrm{Sp}$ \\
\hline Pulmonaria obscura & 2.6 & - & - & - & - & - & $\mathrm{Sp}$ \\
\hline Gagea minima & - & 17.5 & 2.5 & - & - & - & $\mathrm{Sp}$ \\
\hline Corydalis cava & 15 & - & 62.5 & - & - & - & $\mathrm{Sp}$ \\
\hline Adoxa moschatellina & 15 & - & 2.5 & - & - & - & $\mathrm{Sp}$ \\
\hline Viola reichenbachiana & - & - & 0.2 & - & - & - & $\mathrm{Sp}$ \\
\hline Dryopteris filix-mas & - & - & 0.1 & - & - & - & $\mathrm{Sp}$ \\
\hline Atrichum undulatum d & - & - & 0.1 & - & - & - & - \\
\hline \multicolumn{8}{|l|}{ ChCl. Querco-Fagetea } \\
\hline Acer platanoides $\mathrm{a}_{1}$ & - & 87.5 & 37.6 & - & - & - & \\
\hline Acer platanoides $\mathrm{a}_{2}$ & - & 2.5 & 2.5 & - & - & - & An \\
\hline Acer platanoides $\mathrm{b}$ & 5 & 5 & 20 & - & - & - & 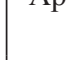 \\
\hline Acer platanoides $\mathrm{c}$ & 2.6 & 17.5 & 55.1 & 2.5 & 2.5 & - & \\
\hline Fraxinus excelsior $\mathrm{a}_{1}$ & 15.1 & 77.5 & 15 & - & - & - & \\
\hline Fraxinus excelsior $\mathrm{a}_{2}$ & - & 15 & - & - & - & - & An \\
\hline Fraxinus excelsior b & - & - & - & 2.5 & - & - & \\
\hline Fraxinus excelsior c & 2.5 & 0.1 & 7.5 & 0.1 & 2.5 & - & \\
\hline Euonymus europaea $\mathrm{b}$ & - & 17.5 & - & 15 & - & - & \\
\hline Euonymus europaea c & 0.1 & 5 & 5.2 & 2.5 & - & - & Ap \\
\hline Corylus avellana $\mathrm{c}$ & - & 0.1 & - & - & - & - & $\mathrm{Sp}$ \\
\hline Anemone nemorosa & 17.5 & - & 17.5 & - & - & - & $\mathrm{Sp}$ \\
\hline Lonicera xylosteum b & - & - & 20 & - & - & - & $\mathrm{S}$ \\
\hline Lonicera xylosteum c & - & - & 5 & - & - & - & $\mathrm{sp}$ \\
\hline Poa nemoralis & - & - & 2.5 & - & - & - & $\mathrm{Sp}$ \\
\hline Viola riviniana & - & - & 0.1 & - & - & - & $\mathrm{Sp}$ \\
\hline \multicolumn{8}{|l|}{ ChCl. Rhamno-Prunetea } \\
\hline Sambucus nigra $\mathrm{b}$ & 150 & 77.5 & 152.6 & - & - & - & An \\
\hline Sambucus nigra c & 5 & 7.5 & 10 & - & - & - & \\
\hline Clematis vitalba $\mathrm{b}$ & - & - & - & 15 & - & - & $K_{0}$ \\
\hline Clematis vitalba $\mathrm{c}$ & - & 2.5 & 15 & 2.5 & - & - & $\mathrm{Kn}$ \\
\hline \multicolumn{8}{|c|}{ ChCl. Artemisietea vulgaris } \\
\hline Aegopodium podagraria & 125 & 190 & 175 & - & 15 & 150 & Ap \\
\hline Urtica dioica & 0.1 & 0.2 & - & - & 0.1 & 90 & Ap \\
\hline Geranium robertianum & 2.6 & - & 5 & - & - & - & Ap \\
\hline Geum urbanum & 2.5 & 2.6 & 0.1 & 0.1 & - & - & Ap \\
\hline
\end{tabular}




\begin{tabular}{|c|c|c|c|c|c|c|c|}
\hline Chelidonium majus & - & 2.6 & 2.6 & - & - & - & Ap \\
\hline Galium aparine & - & 5 & 10 & - & - & - & Ap \\
\hline Viola odorata & - & 17.5 & - & - & 0.1 & - & Ap \\
\hline Galeopsis pubescens & 0.1 & - & 0.1 & - & - & - & Ap \\
\hline Glechoma hederacea & - & 2.5 & - & - & 15.1 & 5 & Ap \\
\hline Chaerophyllum temulum & - & 2.5 & 0.1 & - & - & - & $\mathrm{Sp}$ \\
\hline Anthriscus sylvestris & - & 0.1 & 2.5 & - & 0.1 & 17.5 & Ap \\
\hline Impatiens parviflora & - & - & 15 & - & - & - & $\mathrm{Kn}$ \\
\hline Lamium album & - & - & - & - & 2.6 & 2.5 & $\mathrm{Ar}$ \\
\hline Equisetum arvense & - & - & - & - & 2.5 & 0.1 & Ap \\
\hline Arctium lappa & - & - & - & - & 0.1 & - & Ap \\
\hline Artemisia vulgaris & - & - & - & - & 2.5 & - & Ap \\
\hline Tanacetum vulgare & - & - & - & - & 2.5 & - & Ap \\
\hline Impatiens glandulifera & - & - & - & - & - & 2.5 & $\mathrm{Kn}$ \\
\hline Calystegia sepium & - & - & - & - & - & 2.5 & Ap \\
\hline \multicolumn{8}{|l|}{ ChO. Arrhenatheretalia } \\
\hline Arrhenatherum elatius & - & - & - & 2.5 & 87.5 & 17.5 & Ap \\
\hline Achillea millefolium & - & - & - & - & 17.5 & 0.1 & Ap \\
\hline Dactylis glomerata & - & - & - & 0.1 & 17.5 & 0.1 & Ap \\
\hline Taraxacum officinale coll. & - & 0.1 & - & - & 2.5 & 2.5 & Ap \\
\hline Tragopogon pratensis & - & - & - & - & 0.1 & - & Ap \\
\hline \multicolumn{8}{|c|}{ ChCl. Molinio-Arrhenatheretea } \\
\hline Rumex acetosa & - & - & - & - & 2.5 & - & Ap \\
\hline Carex hirta & - & - & - & 2.5 & 0.1 & - & Ap \\
\hline Lathyrus pratensis & - & - & - & - & 0.1 & - & $\mathrm{Sp}$ \\
\hline Lolium perenne & - & - & - & - & 2.5 & - & Ap \\
\hline Phleum pratense & - & - & - & - & 2.5 & - & Ap \\
\hline Plantago lanceolata & - & - & - & - & 2.5 & - & Ap \\
\hline Poa pratensis & - & - & - & 0.1 & 2.5 & 2.5 & Ap \\
\hline Ranunculus repens & 0.1 & - & - & - & 2.5 & - & Ap \\
\hline Trifolium repens & - & - & - & - & 15 & - & Ap \\
\hline Trifolium pratense & - & - & - & - & - & 0.1 & Ap \\
\hline Veronica chamaedrys & - & 2.5 & 0.1 & 0.1 & 2.6 & 0.1 & $\mathrm{Sp}$ \\
\hline Ranunculus acris & - & - & - & 2.5 & 0.1 & 2.5 & Ap \\
\hline Poa trivialis & - & - & - & - & - & 2.5 & Ap \\
\hline Stellaria graminea & - & - & - & - & - & 0.1 & $\mathrm{Sp}$ \\
\hline
\end{tabular}




\begin{tabular}{|c|c|c|c|c|c|c|c|}
\hline \multicolumn{8}{|l|}{ ChCl. Stellarietea mediae } \\
\hline Stellaria media & - & - & - & - & 2.5 & 2.5 & Ap \\
\hline Geranium pusillum & - & - & - & - & 0.1 & - & $\mathrm{Ar}$ \\
\hline Lamium purpureum & - & - & - & - & - & 2.5 & $\mathrm{Ar}$ \\
\hline Atriplex patula & - & - & - & - & - & 0.1 & Ap \\
\hline Lapsana communis & - & - & - & - & - & 0.1 & Ap \\
\hline \multicolumn{8}{|c|}{ ChCl. Agropyretea intermedio-repentis } \\
\hline Convolvulus arvensis & - & - & - & - & 2.5 & - & Ap \\
\hline Elymus repens & - & - & - & - & 2.5 & - & Ap \\
\hline Poa angustifolia & - & - & - & - & 2.5 & - & Ap \\
\hline \multicolumn{8}{|l|}{ ChCl. Nardo-Callunetea } \\
\hline Agrostis vulgaris & - & - & - & - & 15 & 2.5 & Ap \\
\hline Carex pilulifera & - & - & - & 2.5 & - & - & Ap \\
\hline Danthonia decumbens & - & - & - & - & 2.5 & - & $\mathrm{Sp}$ \\
\hline \multicolumn{8}{|l|}{ Others } \\
\hline Aesculus hippocastanum $\mathrm{a}_{1}$ & 37.5 & - & - & - & - & - & \\
\hline Aesculus hippocastanum $\mathrm{a}_{2}$ & 15 & - & - & - & - & - & $\mathrm{Kn}$ \\
\hline Aesculus hippocastanum c & 2.5 & - & - & - & - & - & \\
\hline Aesculus hippocastanum b & - & 2.5 & - & - & - & - & \\
\hline Quercus robur $\mathrm{a}_{1}$ & - & 0.1 & 5 & - & - & - & \\
\hline Quercus robur b & - & - & - & 2.5 & - & - & $\mathrm{Sp}$ \\
\hline Quercus robur c & - & 0.2 & 0.1 & - & 2.5 & 0.1 & \\
\hline Picea abies $\mathrm{a}_{2}$ & - & 15 & - & - & - & - & $\mathrm{Sp}$ \\
\hline Symphoricarpos albus b & - & 5 & - & - & - & - & $\mathrm{Kn}$ \\
\hline Hedera helix $\mathrm{b}$ & - & - & 2.5 & - & - & - & $\mathrm{Sn}$ \\
\hline Hedera helix $\mathrm{c}$ & 15 & 15 & 102.5 & - & - & - & $\mathrm{sp}$ \\
\hline Ribes uva-crispa c & 2.5 & 2.5 & - & - & - & - & $\mathrm{Kn}$ \\
\hline Prunus cerasifera $\mathrm{c}$ & 0.1 & - & - & - & - & - & $\mathrm{Kn}$ \\
\hline Betula pendula $\mathrm{a}_{1}$ & - & - & 15 & - & - & - & Ap \\
\hline Robinia pseudoacacia $\mathrm{a}_{1}$ & - & - & 15 & - & - & - & $\mathrm{Kn}$ \\
\hline Ribes rubrum b & - & - & 0.1 & - & - & - & $\mathrm{Kn}$ \\
\hline Parthenocissus inserta $\mathrm{b}$ & - & - & - & 87.5 & - & - & $\mathrm{Kn}$ \\
\hline Parthenocissus inserta c & - & - & - & 15 & - & - & \\
\hline Veronica hederifolia & - & 30 & 2.5 & - & - & - & Ap \\
\hline Moehringia trinervia & - & 2.5 & 2.6 & - & - & - & $\mathrm{Sp}$ \\
\hline Oxalis acetosella & 2.5 & - & - & - & - & - & Ap \\
\hline
\end{tabular}




\begin{tabular}{|c|c|c|c|c|c|c|c|}
\hline Dryopteris dilatata & 0.1 & - & - & - & - & - & $\mathrm{Sp}$ \\
\hline Dryopteris carthusiana & 0.1 & - & - & - & - & - & $\mathrm{Sp}$ \\
\hline Astragalus glycyphyllos & - & - & - & 15 & - & - & $\mathrm{Sp}$ \\
\hline Galium album & - & - & - & 2.5 & 15 & - & Ap \\
\hline Medicago sativa & - & - & - & - & 2.5 & - & $\mathrm{Kn}$ \\
\hline Galium verum & - & - & - & - & 2.5 & - & $\mathrm{Sp}$ \\
\hline Prunus domestica & - & - & - & - & 0.1 & - & $\mathrm{Kn}$ \\
\hline Capsella bursa-pastoris & - & - & - & - & 0.1 & - & $\mathrm{Ar}$ \\
\hline Conyza canadensis & - & - & - & - & 0.1 & - & $\mathrm{Kn}$ \\
\hline Erigeron anпuиs & - & - & - & - & 2.5 & 2.5 & $\mathrm{Kn}$ \\
\hline Plantago major & - & - & - & - & 2.5 & - & Ap \\
\hline Verbascum nigrum & - & - & - & - & 2.5 & - & $\mathrm{Sp}$ \\
\hline Symphytum officinale & - & - & - & - & - & 0.1 & $\mathrm{Sp}$ \\
\hline Brachythecium rutabulum $\mathrm{d}$ & 2.5 & 2.7 & 0.1 & - & 2.5 & 5 & - \\
\hline Eurhynchium hians $\mathrm{d}$ & 0.1 & 2.6 & - & - & - & 0.1 & - \\
\hline Hypnum cupressiforme $\mathrm{d}$ & 0.1 & - & - & - & - & - & - \\
\hline Plagiomnium cuspidatum d & - & 0.1 & - & - & - & - & - \\
\hline Brachythecium velutinum d & - & - & 0.1 & - & - & - & - \\
\hline
\end{tabular}

elatius and other meadows species are fairly numerous apart from them.

Species belonging to Querco-Fagetea class are dominant in associations: Fraxino-Alnetum - 19 species (54\%) and Tilio-Carpinetum - 24 species (54\%). In Ficario-Ulmetum the share of species from this class is smaller and amounts to $39 \%$ (15 taxa). Non-forest species, mainly from Artemisietea vulgaris class have a large share in all localities (Fig. 3).

In non-forest vegetation of the park species characteristic for these units are dominant. In Arrhenatheretum elatioris, aside from 16 species of Molinio-Arrhenetheretea class $(33 \%)$, species from six other classes are present, including the most numerous of Artemisietea vulgaris class (10\%). Species of Molinio-Arrhenatheretea, Stellarietea mediae and Nardo-Callunetea classes occur in Urtico-Aegopodietum, apart from the species of Artemisietea vulgaris class (36\%) (Fig. 4).

The index of natural character $(\mathrm{N})$ of the forest communieties is: $40.00 \%$ for Ficario-Ulmetum, $46.87 \%$ for Fraxino-Alnetum and $51.22 \%$ for Tilio-Carpinetum (Table 2). This index is lower in non-forest communities, ranging from 12.76\% (Arrhenatheretum elatioris) to
18.75\% (Parthenocissus inserta and Clematis vitalba community). Synanthropization index among forest community reached the lowest value for Tilio-Carpinetum $(48.76 \%)$ and the highest for Ficario-Ulmetum (60.00\%) This index is over $80 \%$ for non-forest communities. The value of the synanthropization index in forest communities is influenced by the presence of kenophytes: Aesculus hippocastanum, Clematis vitalba, Impatiens parviflora, Prunus cerasifera, Ribes rubrum, Ribes uvacrispa, Robinia pseudoacacia, Symphoricarpos albus. Tilio-Carpinetum had the lowest value of apophytization index (39.02\%) and Arrhenatheretum elatioris the highest $(72.34 \%)$.

Associations Tilio-Carpinetum, Ficario-Ulmetum, Fraxino-Alnetum and Arrhenatheretum elatioris represents 4 types of Natura 2000 sites - respectively type: 9170, *91E0, 91F0, 6510 (Herbich, 2004a, b).

\section{Discussion}

The current state of the vegetation in the park in Laskowice results mainly from diversified habitat condition 
(lie of the land, lake vicinity) and lack of maintenance causing progression of regeneration. As in case of other non-maintained parks with a landscape character there occurs a restoration of forest communities in accordance with the habitat. Tilio-Carpinetum occupies the greatest area in the park. It is the most often documented community of non-maintained village parks (Fornal-Pieniak \& Wysocki, 2006; Fornal-Pieniak \& Ollik, 2013; Kucharski \& Chmielec- ki, 2014. Ficario-Ulmetum (Kułak \& Sender, 2011) and Fraxino-Alnetum (Borysiak et al., 2011a; Sobisz \& Szmyt, 2015) are mentioned uncommonly.

The plant communities occurring in the park in Laskowice may be determined through the presence of characteristic species. An attention should be paid on physiognomy and species composition of forest communities diverging from typical forms. A strongly developed shrub layer brings attention primarily. There is a lack of some charac-
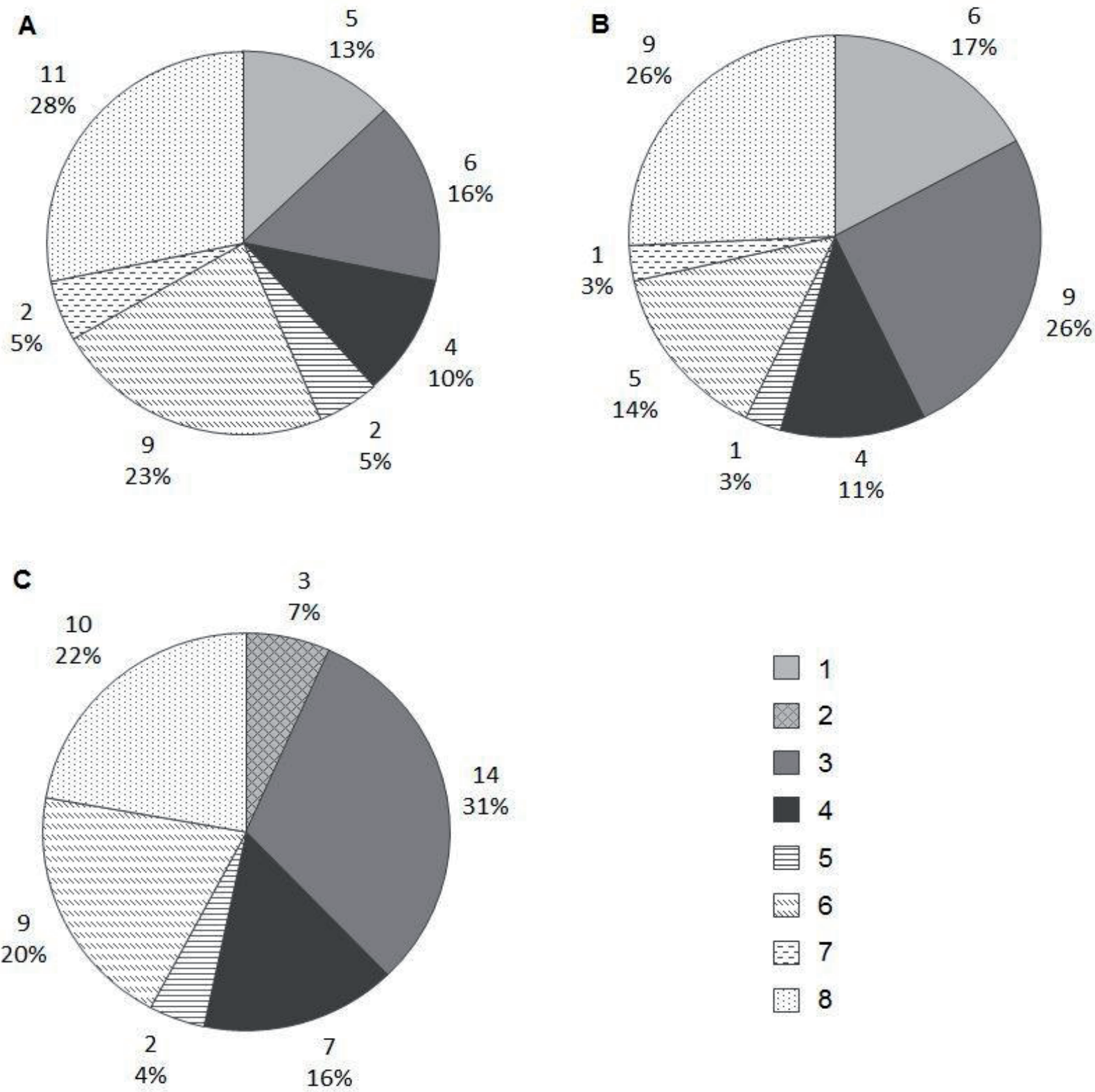

Figure 3. Number and percentage share of species from phytosociological classes in forest plant communities (A - Ficario-Ulmetum, B - Fraxino-Alnetum, C - Tilio-Carpinetum; 1 - Alno-Ulmion, 2 - Carpinion betuli, 3 - Fagetalia sylvaticae, 4 - QuercoFagetea, 5 - Rhamno-Prunetea, 6 - Artemisietea vulgaris, 7 - Molinio-Arrhenatheretea, 8 - others) 

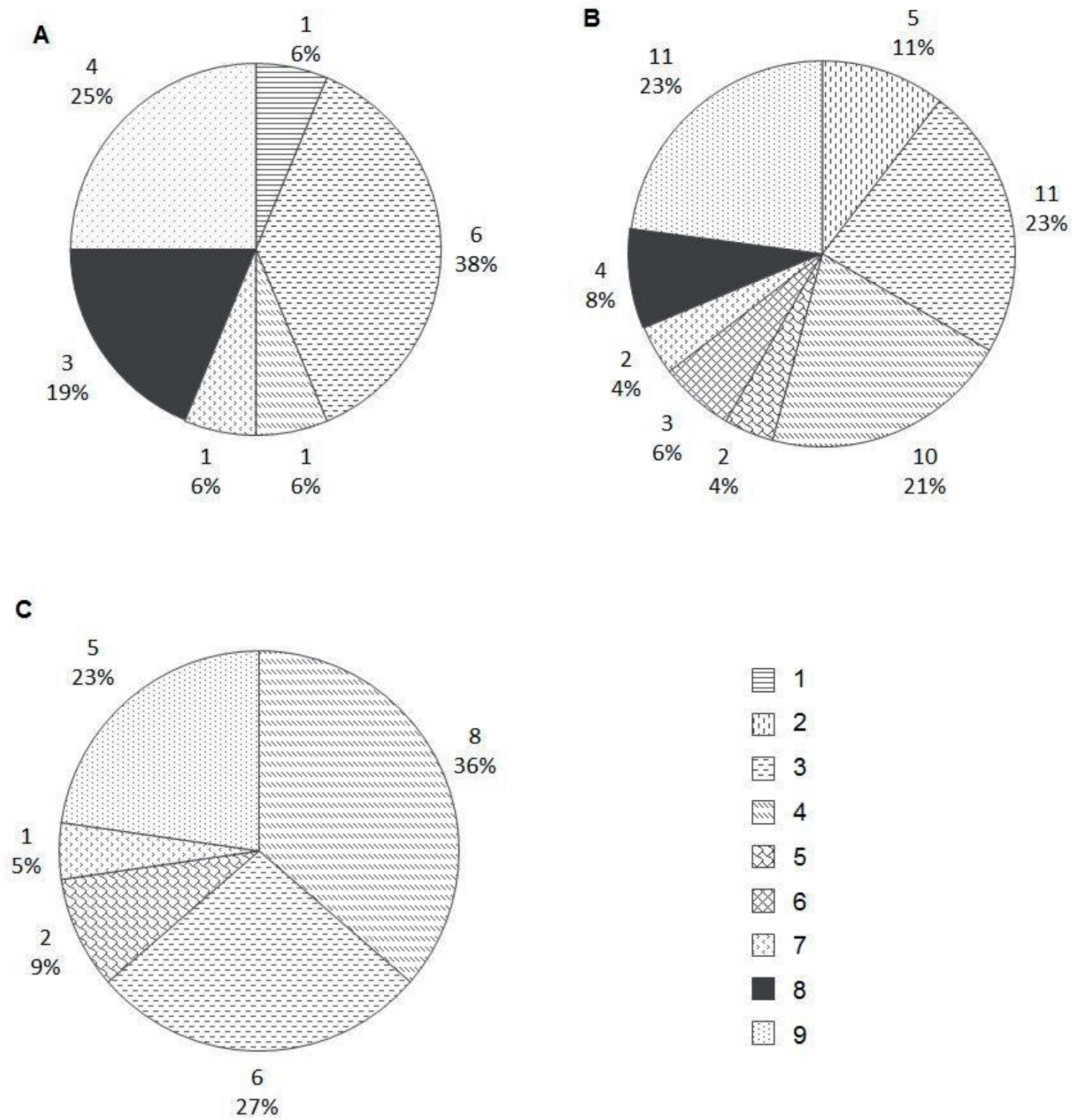

Figure 4. Number and percentage share of species from phytosociological classes in non-forest plant communities (A - Parthenocissus inserta and Clematis vitalba community, B - Arrhenatheretum elatioris, C - Urtico-Aegopodietum; 1 - Rhamno-Prunetea, 2 - Arrhenatheretalia, 3 -Molinio-Arrhenatheretea, 4 -Artemisietea vulgaris, 5 - Stellarietea mediae, 6 - Agropyretea intermedio-repenti, 7 - Nardo-Callunetea, 8 - Querco-Fagetea, 9 - others)

Table 2. Anthropogenic indices of plants communities (F-A - Fraxino-Alnetum, F-U - Ficario-Ulmetum minoris, T-C - Tilio-Carpinetum, Par.-Cle. - Parthenocissus inserta and Clematis vitalba community, Arr. - Arrhenatheretum elatioris, U-A - UrticoAegopodietum)

\begin{tabular}{|l|c|c|c|c|c|c|}
\hline \multicolumn{1}{|c|}{ Indices } & F-A & F-U & T-C & Par.-Cle. & Arr. & U-A \\
\hline Index of natural character (\%) & 46.87 & 40.00 & 51.22 & 18.75 & 12.76 & 15.38 \\
\hline Synanthropization index (\%) & 53.53 & 60.00 & 48.78 & 81.25 & 87.23 & 84.61 \\
\hline Apophytization index (\%) & 43.75 & 48.57 & 39.02 & 68.75 & 72.34 & 69.23 \\
\hline
\end{tabular}


teristic species and geographically alien species are present in treestand and shrub layer.

Phytocoenoses Ficario-Ulmetum and Tilio-Carpinetum are characterized with a large share of Sambucus nigra and in case of the first association also Philadelphus coronarius, Symphoricarpos albus and Aegopodium podagraria in a herb layer. Other objects have also a similar character. The analysis of vegetation of village parks of Sandomierz Basin allowed to distinguish Tilio-Carpinetum association i.a. in two forms - with a small share of distinguishing species and Tilio-Carpinetum with Sambucus nigra and Aegopodium podagraria (Fornal-Pieniak \& Wysocki, 2006). Dombrowicz (1994) paid attention on fruticetisation (i.a. by Sambucus nigra), as a main form of degeneration of village parks near Bydgoszcz. Sambucus nigra and Philadelphus coronarius are also the most frequently occurring shrubs in manor park in Sobianowice (Pudelska \& Biesak, 2016). It should be underlined, that fruticetisation is one on the main forms of degeneration of linden-oak-hornbeam forests in Poland and Rubus are yet common taxa causing development of shrubs (Woziwoda, 2007).

The presence of shrub communities of Rhamno-Prunetea class is typical for overgrowing parks. Parthenocissus inserta and Clematis vitalba community developed on the edges of the park ruins. No information about the presence of this community was found in literature, although the occurrence of both species in other manor parks was noted (Kucharski \& Chmielecki, 2014; Sobisz \& Szmyt, 2015).

Meadow communities from Molinio-Arrhenatheretea class and ruderal from Artemisietea vulgaris occupy a small area in the park and they were documented from places, which during functioning of the palace and park were deprived of trees. It is a fragment of the park by the entrance gate, where a tennis court was probably located and subsequently a playground. The second non-forest place is a slope, where the palace was erected. A mosaic of communities from these two classes has currently developed in that place. Non-forest vegetation of the park is less degraded than forest vegetation and has a more typical species composition (Matuszkiewicz, 2001). Non-forest communities are rarely described from manor complexes (Kułak \& Sender, 2011; Borysiak et al., 2011a).

The values of the index of natural character and the synanthropization index of the forest community in manor park in Laskowice indicate their regeneration, although the presence of alien species still remains. The index of natural character of forest community of manor park in Laskowice is $\geq 40 \%$. In comparison, this indicator calculated for NE Wielkopolska reserves is 45.5\% (Kutyna et al., 2013). Tilio-Carpinetum has the lowest synanthropization index (48.78\%), and Ficario-Ulmetum the highest (60.00\%). For comparison, the synanthropisation index of forest habitats distant about $10 \mathrm{~m}$ from the mid-forest car parks in the
Wkrzańska and Bukowa Primeval Forests is $68.8 \%$ for coniferous habitats and $66.1 \%$ for forest habitats (Kutyna et al., 2010). The lower values of the index of natural character and the higher index of synanthropization in the non-forest communities are due to their anthropogenic syngenesis. Synanthropization index of Arrhenatheretum elatioris of manor park is $87.23 \%$. A similar value of this index $(81.0 \%)$ was obtained for the same community occurring in meadows in the larger Wielkopolska river valleys (Kryszak, 2004).

A large share of ruderal species in manor park in Laskowice may result from a high habitat trophy due to build-up rural areas and the presence of numerous dirt paths, frequented by man (Latowski \& Zieliński, 2001). In village parks an abundance of Aegopodium podagraria is common. Its large share was also noted in park ruins in Granowo (Borysiak et al., 2011b) and in village parks of Sandomierz Basin (Fornal-Pieniak \& Wysocki, 2006).

Neglected manor parks in rural landscape constitute environmentally valuable ecological islands (Latowski \& Zieliński, 2001) and their unquestionable quality is an occurrence of Natura 2000 sites (Borysiak et al., 2011a).

Revitalization activities are planned in manor park in Laskowice, aiming at restoration of recreational function of this place, but with preserving the current vegetation character. The removal of a shrub layer is planned in phytocoenosis of Ficario-Ulmetum. Phytocoenosis of Tilio-Carpinetum is planned to be left with no activities but with keeping the existing walking alleys. Proceeding natural regeneration of these communities with concurrent, minimally invasive revitalization activities, gives an opportunity to improve the state of deformed forest phytocoenoses. Whereas non-forest phytocoenoses with an anthropogenic derivation are suggested to be regularly mowed (Paszek \& Gawenda-Kempczyńska, 2016).

\section{References}

Bacieczko W. \& Wołejko L., 2011, Aktualny stan flory i znaczenie osobliwości florystycznych dla różnorodności szaty roślinnej rezerwatu „Grądowe Zbocza" koło Recza [Present state of flora and importance of floristic rarities for diversity of vegetation cover of the nature reserve „Grądowe Zbocza” near Recz], Folia Pomer. Univ. Technol. Stetin., Agric., Aliment., Pisc., Zootech. 289(19): 7-26.

Borowski J., Sikorski P., Wierzba M. \& Wysocki Cz., 2007, Metody inwentaryzacji roślinności w parkach zabytkowych o charakterze krajobrazowym na podstawach geobotanicznych [Methods of vegetation inventory-making in historical landscape park based on geobotanical knowledge], Sylwan 12: 30-39. 
Borysiak A., Borysiak J. \& Markiewicz J., 2011a, Naturalistyczny park w Ciążeniu w ochronie siedlisk przyrodniczych Natura 2000 obszaru PLH300009 Ostoja Nadwarciańska [Naturalistic park in Ciążeń in the Natura 2000 habitat protection of the site PLH 300009 Ostoja Nadwarciańska], Bad. Fizjogr., R. II (B60): 33-60.

Borysiak A., Borysiak J., Dreszer W. \& Dreszer S., 2011b, Koncepcja rewaloryzacji naturalistycznego parku w Granowie w województwie wielkopolskim [Concept of revalorisation of a naturalistic park in Granowo (Wielkopolska region)], Bad. Fizjogr., R. II (B60): 7-32.

Chmiel., 1993, Flora roślin naczyniowych wschodniej części Pojezierza Gnieźnieńskiego i jej antropogeniczne przeobrażenia w wieku XIX i XX. Część 1 [Flora of vascular plants of the eastern part of the Gniezno Lake District and its transformation under the influence of man in 19th and 20th centuries. Part 1], Prace Zakładu Taksonomii Roślin UAM w Poznaniu, Sorus, Poznań.

Chudecki M., 1994, Zapomniany park [Forgotten park], Echo Borów Tucholskich 6(33): 10-11.

Didyk K., 2013 mscr., Walory przyrodnicze i wstępna inwentaryzacja parku podworskiego w Laskowicach, Praca inżynierska [Nature conservation values and preliminary inventory of the manor park in Laskowice, Engineering thesis], Katedra Botaniki i Ekologii Uniwersytet Techniczno-Przyrodniczy w Bydgoszczy, Bydgoszcz.

Dombrowicz M., 1994, Formy degeneracji leśnych zbiorowisk roślinnych w parkach wiejskich województwa bydgoskiego [Degradation forms of forest plant communities in rural parks of the Bydgoskie voivodeship], Zeszyty Naukowe WSP w Bydgoszczy, Studia Przyrodnicze 10: 73-78.

Dzwonko 2007., Przewodnik do badań fitosocjologicznych [Guidebook to phytosociological studies], Sorus, Instytut Botaniki Uniwersytetu Jagiellońskiego, Poznań Kraków.

Fornal-Pieniak B. \& Wysocki Cz., 2006, Flora structure in rural parks on example of Sandomierska Valley, Acta Sci. Pol. Silv. Colendar. Rat. Ind. Lignar. 5(2): 31-45.

Fornal-Pieniak B. \& Ollik M., 2013, Diversity of flora in the undergrowth of park afforestations, rural plantings and oak-hornbeam forests, Folia Forestalia Polonica, Series A, 55 (3): 132-136.

Herbich J., (ed.), 2004a, Lasy i bory. Poradniki ochrony siedlisk i gatunków Natura 2000 - podręcznik metodyczny, T. 5, Ministerstwo Środowiska, Warszawa [Deciduous and coniferous forests, Vol. 5, Natura 2000 habitat and species protection guides - methodological handbook, Ministry of the Environment, Warsaw].

Herbich J., (ed.), 2004b, Murawy, łąki, ziołorośla, wrzosowiska, zarośla, T. 3, Poradniki ochrony siedlisk i gatunków Natura 2000 - podręcznik metodyczny, Mini- sterstwo Środowiska, Warszawa, [Grasslands, meadows, tall-herb vegetation, heaths, scrubs, Vol. 3, Natura 2000 habitat and species protection guides - methodological handbook, Ministry of the Environment, Warsaw].

http://amzpbig.com/maps/2476_Jaschewo_1910.jpg.

Kryszak A., 2004, Synantropizacja wybranych zbiorowisk łąkowych [Synanthropisation of selected meadow communities], Woda - Środowisko - Obszary Wiejskie 4, 1(10): 201-208.

Kucharski L. \& Chmielecki B., 2014, Dendroflora parku zamkowego w Uniejowie - stan obecny i zmiany [Dendroflora of the castle park in Uniejów - current condition and changes], Biuletyn Uniejowski 3: 5-22.

Kułak A. \& Sender J., 2011, Park-and-palace complexes as biodiversity refuges on example Gałęzów ex-manor park, Teka Kom. Ochr. Kszt. Środ. Przyr. - OL PAN 8: 73-85.

Kutyna I. \& Malinowska K., 2010, Struktura geograficzno-historyczna flory oraz jej stopień synantropizacji w fitocenozach leśnych przylegających do parkingów oraz występujących w ich obrębie [Geographic and historical structure of flora and its degree of synanthropy in forest phytocenoses adjacent to car parks and found in their neighbourhood], Folia Pomer. Univ. Technol. Stetin., Agric., Aliment., Pisc., Zootech. 279(15): 45-52.

Kutyna I., Berkowska E. \& Młynkowiak E., 2013, Struktura geograficzno-historyczna flor zróżnicowanych biotopów oraz wybrane wskaźniki antropogeniczne [The geographical and historical structure of floras of differentiated biotopes and the selected anthropogenic indices], Folia Pomer. Univ. Technol. Stetin., Agric., Aliment., Pisc., Zootech. 302(25): 95-112.

Latowski K. \& Zieliński J., 2001, Parki wiejskie wybrane zagadnienia geobotaniczne i kulturowe [Village parks - selected geobotanical and culture problems], [in:] Szata roślinna Wielkopolski i Pojezierza Południowopomorskiego. Przewodnik sesji terenowych 52. Zjazdu PTB 24-28 września 2001 [Vegetation cover of the Wielkopolska Region and the South Pomeranian Lake District, Guidebook to field sessions of the 52nd Polish Botanical Society Convention, 24-28 September, 2001], M. Wojterska (ed.), Bogucki Wyd. Nauk., Poznań: 39-110.

Matuszkiewicz W., 2001, Przewodnik do oznaczania zbiorowisk roślinnych Polski [Guide for the determination of Polish plant communities], Wyd. Nauk. PWN, Warszawa.

Mirek Z., Piękoś-Mirkowa H., Zając A. \& Zając M., 2002, Flowering plants and pteridophytes of Poland. A checklist, W. Szafer Ins. Bot. Pol. Acad. Sci., Kraków.

Obwieszczenie M.Ś. z dn. 30 października 2014, Poz. 1713, w sprawie ogłoszenia jednolitego tekstu 
rozporządzenia Ministra Środowiska w sprawie siedlisk przyrodniczych oraz gatunków będących przedmiotem zainteresowania Wspólnoty, a także kryteriów wyboru obszarów kwalifikujących się do uznania lub wyznaczenia jako obszary Natura 2000 [Notice of the Minister of the Environment dated 30 October 2014 on the publication of the consolidated text of the Regulation of the Minister of the Environment on natural habitats and species of the Community interest, and criteria for selection of areas eligible for recognition or designation as Natura 2000 sites], Dz. U. RP 2014, Poz. 1713, Kanc. Prezesa Rady Min. [Journal of Laws RP 2014, item 1713, Chancellery of the Prime Minister], 2014, Warszawa.

Paszek I. \& Gawenda-Kempczyńska D., 2016 mscr., Szata roślinna parku dworskiego w Laskowicach [Plant cover of manor park in Laskowice], VITIS Iwona Paszek, Bydgoszcz.

Pietrzak J. \& Zawadka J., 2009, Wykorzystanie turystyczne drzew pomnikowych na terenie Lasów Państwowych [The use of tourist of monumental trees of The State Forests National Forest Holding territory], Studia i Materiały Centrum Edukacji Przyrodniczo-Leśnej 11, 4(23): 158-164.

Pudelska K. \& Biesak A., 2016, Analiza dendroflory parku dworskiego w Sobianowicach (woj. lubelskie) [Analysis of the dendroflora in the manor park in Sobianowice
(Lublin voivodeship)], Nauka Przyroda Technologie 10(3, 38): 1-14.

Sobisz Z. \& Szmyt E., 2015, Park podworski w Jaworach interesujący obiekt przyrodniczy Parku Krajobrazowego „Dolina Słupi” [Former manor park in Jawory - an interesting part of Słupia River Valley Landscape Park], Słupskie Prace Biologiczne 12: 187-208.

Tokarska-Guzik B., Dajdok Z., Zając M., Zając A., Urbisz A., Danielewicz W. \& Hołdyński C., 2012, Rośliny obcego pochodzenia w Polsce ze szczególnych uwzględnieniem gatunków inwazyjnych [Alien plants in Poland with particular reference to invasive species], Generalna Dyrekcja Ochrony Środowiska, Warszawa. von Weitzel-Zenker A., 2002, Am Ufer der Erinnerung. Landsberg, Selbstverlag.

Wildner-Nurek I., 2010, Lista preferencyjna zabytkowych parków i ogrodów dla Narodowego Funduszu Ochrony Środowiska i Gospodarki Wodnej - podsumowanie dotychczasowych doświadczeń, Kurier konserwatorski 7: 61-68.

Woziwoda B., 2007, Identyfikacja i ocena stanu zachowania siedlisk grądowych (kod Natura 2000: 9170) w lasach Polski środkowej [Identification and evaluation of the oak-lime - hornbeam forests condition (9170 - the Nature 2000 code) in central Poland], Studia i Materiały Centrum Edukacji Przyrodniczo-Leśnej 9, 2/3(16): 59-69. 